\title{
ESTRATEGIA DE MANEJO DE LOS RECURSOS MARINOS Y COSTEROS EN ISLA UVITA, LIMÓN, COSTA RICA
}

\author{
José Pereira Chaves ${ }^{1 *}$ y Luis Sierra Sierra ${ }^{2}$
}

\begin{abstract}
RESUMEN
El presente trabajo se realizó en la Isla Uvita, declarada Monumento Nacional en 1985, ubicada en el litoral Caribe de Costa Rica. Esta zona se caracteriza por tener una gran riqueza de sus recursos marinos y costeros. El propósito fundamental fue ofrecer diversas acciones de conservación, manejo y desarrollo sostenible en dicha área, promoviendo la coordinación y participación de todos los sectores claves. Se hace referencia a la necesidad de ejecutar una estrategia de manejo que promueva la estabilidad de los recursos marino-costeros del sitio insular, para fortalecer tanto la riqueza particular como para establecer normas educativas y científicas que promuevan las condiciones adecuadas para el desarrollo sostenible. Isla Uvita presenta problemas de conservación y manejo, debido a la ausencia de una entidad administrativa que controle y norme los usos. Se conoció la percepción social del limonense mediante la aplicación de un cuestionario tipo encuesta y además se desarrolló un taller, en el sitio. Con base en la percepción social, la mayoría opinó que se deben controlar las actividades dentro de la Isla, estableciendo controles de vigilancia, reconocen que este sitio lo debe manejar el MINAET y JAPDEVA, apoyados por la Municipalidad de Limón, la cual ha de adquirir el compromiso de conservación. La estrategia de manejo hace énfasis en seis líneas estratégicas, como la organización institucional, protección, zonificación, educación y capacitación, monitoreo e investigación y recreación.
\end{abstract}

Palabras claves: Manejo, recursos marinos, Limón, Costa Rica.

\begin{abstract}
The Caribbean Coast of Costa Rica is known for its marine resource diversity, and is where the Uvita Island Insular System is located. This site was declared a National Monument in 1985, and chosen as the site for the present study, which had the main objective of offering an adequate managing tool to perform several conservation and sustainable development actions on the Island, to promote the coordination and participation of all stakeholders. The study clearly identified the need for developing a management strategy to promote pertinent conservation actions at the site, to strengthen not only its particular diversity, but also to establish educational and scientific regulations to encourage the adequate conditions for the sustainable development of the Island. Currently, the Island faces conservation and management problems due to the lack of an administrator to control and regulate the use of the site. In order to learn about the perception of the local people, a survey type questionnaire was administered and a workshop held at the site. Based on the results of the survey, the majority of the local people thought that the Island activities should be regulated and vigilance controls established. It was also well recognized that government institutions, such as MINAET and JAPDEVA, should manage the site with the support of the local government, which should acquire a commitment towards

1 Escuela de Ciencias Biológicas, Universidad Nacional, Heredia, Costa Rica. jpereira@una.ac.cr*, 1sierra@una.ac.cr
\end{abstract}

Recibido 26-V-2009

Aceptado 29-IX-2009

DOI: http://dx.doi.org/10.15359/revmar.1.7

Rev. Mar. y Cost. ISSN 1659-455X. Vol. 1. 127-143, Diciembre 2009. 
conservation. The study proposed a management strategy for the Island, placing emphasis on six lines of action: institutional organization, protection, zoning, education and training, monitoring, and research and recreation.

Keywords: Management, marine resource, Limon, Costa Rica.

\section{INTRODUCCIÓN}

En Costa Rica, una de las políticas de la estrategia nacional para la gestión integral de los recursos marinos y costeros es fortalecer y establecer mecanismos técnicos entre el gobierno y la sociedad civil en la gestión integrada de los recursos marinos y costeros (Comisión Interinstitucional de la Zona Económica Exclusiva de Costa Rica, 2008).

Uvita presenta una riqueza histórica, biológica y estructural admirable, posee elementos únicos del Caribe Central, tiene importantes sitios de anidación de aves acuáticas como los piqueros morenos o sulas (Sulas leucogaster), así como importantes formaciones de arrecifes coralinos y esponjas que hacen al sitio un laboratorio viviente.

Isla Uvita no cuenta con ningún régimen de manejo y administración de los recursos, que incentive a los usuarios a la práctica de acciones necesarias para evitar su inadecuado uso. Las principales amenazas corresponden al crecimiento del turismo, donde no hay ningún tipo de planificación y manejo.

El Banco Interamericano de Desarrollo (BID) en 1998 propuso una estrategia para el manejo de los recursos marinos y costeros en América Latina y el Caribe, que se caracterizó no sólo por la representación detallada de una secuencia de accio- nes, sino también por una particular cualidad de dichas acciones.

Aplicando las recomendaciones del BID, se planteó una estrategia de conservación para Isla Uvita, la que presenta pautas para conservar y utilizar sustentablemente la diversidad biológica y establece las prioridades de intervención de los distintos actores claves, así como mantener la integridad y funcionalidad de la biodiversidad.

El propósito de este trabajo fue brindar una herramienta adecuada para guiar las acciones de conservación y desarrollo sostenible del área, promoviendo la coordinación y participación de todos los sectores, por lo que en su desarrollo predominó la directriz de generar un proceso participativo, donde tanto las instituciones gubernamentales, no gubernamentales, comunidades y otros sectores, tuvieran el derecho y el deber de participar en la elaboración, partiendo del conocimiento de la realidad y de los antecedentes del área de estudio.

\section{MATERIALES Y MÉTODOS}

El trabajo se realizó en la Isla Uvita, que está localizada en la provincia de Limón (Figura 1), litoral Caribe de Costa Rica durante el año 2006.

Para conocer la percepción de la población limonense sobre el uso y 
Figura 1

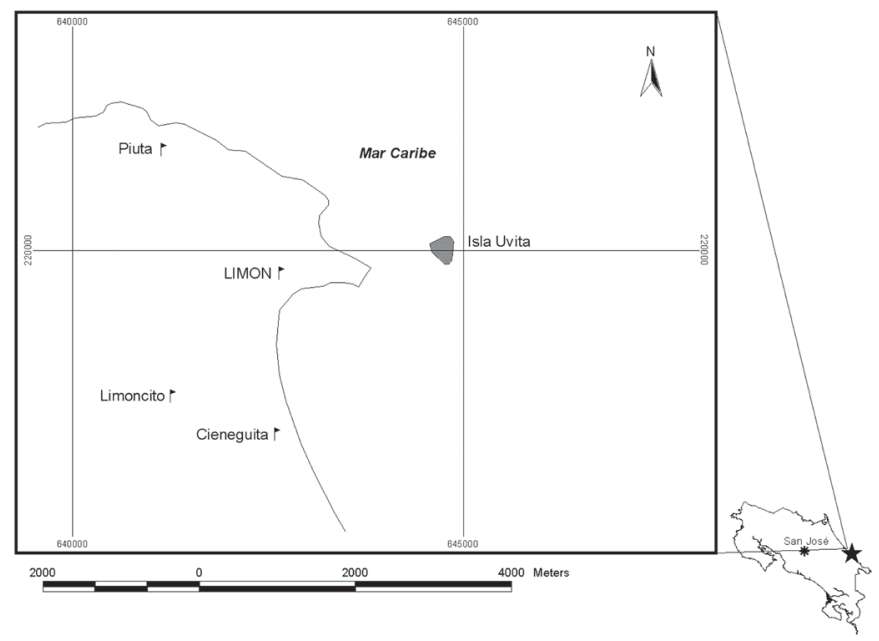

Ubicación geográfica de la Isla Uvita, Limón.

Geographic location of Uvita Island, Limon.

manejo de los recursos marinos y costeros de la Isla, se aplicó una encuesta con preguntas estructuradas abiertas y cerradas, la cual facilitó al encuestado contestar de manera rápida y acorde con los conocimientos que tenían sobre la Isla.

Los muestreos se realizaron en seis segmentos censales propuestos por el Instituto Nacional de Estadística y Censo (INEC), el cual tiene dividida a la ciudad de Limón en nueve segmentos censales (INEC, 2004), los que fueron seleccionados por su cercanía e influencia sobre el sitio de estudio. El tamaño de la muestra se estimó a partir de la fórmula propuesta por Hernández et al. (2006), donde se consideró el nivel de confianza de un $95 \%$, por lo que $Z=1.96$, se estimó un error de muestreo de un 5\%, al aplicar dicha fórmula la muestra correspon- dió a 384 personas, la cual se ajustó a 402 y en cada segmento se le aplicó la encuesta a 67 habitantes de cada segmento censal.

El muestreo realizado fue no probabilístico, debido a que sólo se tomó en cuenta la población de hombres y mujeres mayores de 15 años. Las encuestas se realizaron casa por casa y no se consideró a las personas que no aceptaban o querían responderla, dicho muestreo es considerado estratificado (Gómez, 1998).

Además de las encuestas se realizó un taller para conocer la percepción sobre el futuro uso potencial de la Isla, y obtener información base para la propuesta de la estrategia. La metodología utilizada en el taller fue la propuesta por PROARCA (1998), Ochoa (1998) y Windevoxhel (2001). Los resultados obtenidos en el taller complementaron 
la información obtenida en las encuestas, el cual contó con la participación de representantes de la sociedad civil (pescadores, artesanos, representantes comunales) y algunas instituciones gubernamentales, donde su quehacer tiene cierta relación con el uso de los recursos naturales de la Isla Uvita: (Municipalidad, Ministerio de Ambiente, Energía y Telecomunicaciones (MINAET), Refinadora Costarricense de Petróleo (RECOPE), Ministerio de Educación Pública (MEP) y Junta Administrativa Portuaria de la Vertiente Atlántica (JAPDEVA).

\section{RESULTADOS Y DISCUSIÓN}

La proporción por sexo de las personas encuestadas fue de $1: 1 \mathrm{y} \mathrm{el}$ nivel académico se distribuyó de la siguiente manera: secundaria incompleta un $31 \%$, secundaria completa un $24 \%$, primaria incompleta un $19 \%$, primaria completa un $15 \%$ y universitaria un $11 \%$.

El mayor porcentaje de los(as) ciudadanos(as) encuestados(as) tenían entre 15 y 25 años (32\%) y el menor correspondió a las personas mayores de 56 años (10\%). El 56\% de la población tenía conocimiento de que la Isla es un Monumento Nacional y el $52 \%$ la ha visitado.

Los motivos por los cuales los habitantes visitan la Isla, así como los sitios de mayor preferencia, son para caminar por los senderos y bañarse (Cuadro 1).

\section{Cuadro 1}

Actividades y preferencias de la población que visita la Isla Uvita.

\section{Table 1}

Activities and preferences of the visitors to Uvita Island.

\begin{tabular}{lc|lc}
\hline \multicolumn{2}{c|}{ Actividades } & \multicolumn{2}{c}{ Preferencias } \\
\hline \multicolumn{1}{c}{ Sitios } & Frecuencias & \multicolumn{1}{c}{ Sitios } & Frecuencias \\
1. Caminar & 182 & 1. Sendero & 169 \\
2. Bañarse & 139 & 2. Arrecife & 164 \\
3. Ver el arrecife & 99 & 3. Bosque & 130 \\
4. Bucear/Snorkel & 54 & 4. Peces & 108 \\
5. Pescar & 63 & 5. Playa & 91 \\
6. Surfear & 29 & 6. Aves & 75 \\
\hline
\end{tabular}


Figura 2

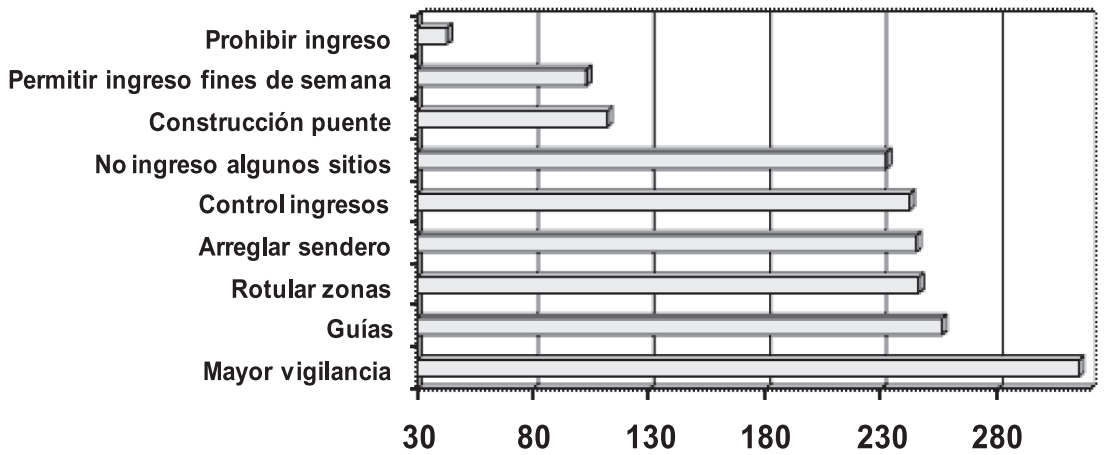

Recomendaciones para minimizar los impactos negativos en Isla Uvita. Limón.

Recommendations for the reduction of negative impacts in Uvita Island. Limon.

Los encuestados consideran que debe haber mayor vigilancia en Isla Uvita (Figura 2), así como el desarrollo de las acciones que minimicen los impactos negativos.

El 86\% de los encuestados recomendó que se debe elaborar e implementar un programa de educación marino-costero, el $75 \%$ consideró que se deben controlar todas las actividades que se desarrollan sobre el arrecife, y el $61 \%$ planteó que se debe realizar una zonificación de la Isla para regular su uso.

El 54\% tenía conocimiento sobre la instalación de un emisario submarino (vertimiento de aguas negras) en los alrededores de la Isla Uvita y de ellos el 92\% opina que el funcionamiento va a causar contaminación marina. Todos afirmaron que si la Isla contara con un plan de manejo o restricciones sería muy beneficioso desde el punto de vista paisajístico, económico, recreativo y ecológico.
La información brindada por los participantes durante el desarrollo del taller realizado permitió conocer la percepción de los actores sobre las amenazas y como ellos perciben el futuro de la Isla Uvita (Cuadro 2).

Los encuestados consideran la Isla como un sitio de interés histórico, con un gran potencial turístico, belleza escénica y que brinda protección a la ciudad de Limón, debido a que por su ubicación geográfica es una barrera de amortiguamiento para la ciudad. El futuro de la Isla lo visualizaron como un área marina protegida, donde se desarrollen actividades de conservación y protección que involucren y comprometan a las autoridades competentes.

Una de las principales preocupaciones de los participantes del taller fue la incertidumbre sobre lo que pueda ocurrir con los recursos marinos y costeros de la Isla. La comunidad limonense no tiene claros los roles que cumplen las instituciones y 


\section{Cuadro 2}

Actividades que generan problemas ecológicos dentro de Isla Uvita, Limón.

Table 2

Activities that generate ecological problems in Uvita Island, Limon.

\begin{tabular}{l|l}
\hline \multicolumn{1}{c|}{ Amenazas } & \multicolumn{1}{c}{ Consecuencias } \\
\hline Ingreso descontrolado de personas. & $\begin{array}{l}\text { Generan desechos inorgánicos, destrucción } \\
\text { y extraen plantas, especies marinas y nidos- } \\
\text { polluelos. } \\
\text { Deteriora el arrecife por el anclaje de botes y } \\
\text { redes sobre él. }\end{array}$ \\
Artes de pesca inapropiadas. & $\begin{array}{l}\text { Las conductas de las personas hacia los recursos } \\
\text { marinos y costeros no son de conservación o } \\
\text { protección sino de destrucción. } \\
\text { Debe haber mayor inversión para que haya más } \\
\text { control sobre la Isla y minimizar los impactos } \\
\text { negativos. } \\
\text { Fay mucha desinformación, no hay señalización } \\
\text { de restricciones a algunos sitios de la Isla, lo que } \\
\text { hace que se utilice toda la porción de tierra. } \\
\text { Va a generar contaminación del agua para los } \\
\text { turistas y acceso de nutrientes marinos. }\end{array}$ \\
Implantación del emisario submarino. & $\begin{array}{l}\text { Ven la Isla como algo para usar y no para } \\
\text { conservar, se sienten dueños de ella pero no la } \\
\text { cuidan. }\end{array}$ \\
\hline Desinterés por parte de la comunidadios y reglamentación.
\end{tabular}

el compromiso de cada una de ellas con la protección de los recursos naturales. No hay una visión clara de lo que se quiere hacer en Uvita. Entre las respuestas obtenidas están: “son muchos los que hablan del sitio pero nadie hace nada". En el debate en el seno del taller por consenso se externó "Que la justificación de la ausencia monetaria no debe ser un pretexto para no conservar la biodiversidad de la Isla, no importa quién la maneje o la administre, mientras la protejan". Sin embargo, el $90 \%$ concuerda con que una de las organizaciones que deberían estar administrando y protegiendo los recursos naturales de la Isla Uvita son: el MINAET, JAPDEVA y la Municipalidad de Limón, en orden de importancia. Estas organizaciones son entidades que han desarrollado diversos proyectos a favor de los servicios comunales y por orden legal deberían ser las que se preocupen por resolver los siguientes problemas que se dan en la Isla: destrucción del arrecife, vertimiento de basura por los visitantes, erosión 
interna, destrucción de sitios de reproducción de aves y la poca capacidad de carga que tiene la Isla, debido a la falta de infraestructura y a condiciones óptimas de senderos y señalamientos del sitio, entre otras.

Entre las sugerencias presentadas por los limonenses para controlar los impactos negativos que se dan sobre la Isla se encuentran:

- Desarrollar programas de monitoreo.

- Establecer programas de educación marino-costera.

- Desarrollar infraestructura adecuada para el visitante.

- Desarrollar programas de voluntariados en la Isla.

- Prohibir a los pescadores que extraigan el recurso dentro de la zona del arrecife.

El trabajo social ha sido importante para conceptualizar y definir los lineamientos ambientales para las actividades que se estarán llevando a cabo en las zonas de influencia de Isla Uvita, como forma de que se minimicen los efectos negativos sobre el medio biofísico y socioeconómico del área.

Considerando que la resiliencia del ecosistema no es solamente amortiguamiento para la perturbación en el sentido de conservar el sistema, es también una fuente de reorganización, renovación, novedad y desarrollo posterior al disturbio, sea este natural o antrópico, mantiene la flexibilidad y oportunidades para responder a cambios ambientales y construir capacidad adaptativa (Gunderson y Pritchard, 2002).

La naturaleza somera del Caribe, su relativo pequeño tamaño y la fuerte influencia continental, la hace altamente vulnerable a los impactos antropogénicos y a las perturbaciones compuestas (Bass et al. 1995).

Se propone un mecanismo para que según el tipo de actividad y su característica se puedan determinar las necesidades y profundidad del desarrollo que se pretende ejecutar en el sitio. Para Windevoxhel (2001), las áreas protegidas cumplen con una gama de papeles definidos, principalmente a través de instrumentos de asignación en diferentes categorías de manejo.

Según Baumgartner (2001) se necesitan estrategias que desarrollen la capacidad para conservar la biodiversidad a largo plazo e incrementar la participación de los actores críticos en la conservación de la biodiversidad. Las medidas del éxito en la conservación evalúan cuán efectivas son las estrategias para mitigar las amenazas y restaurar o mantener la viabilidad de los objetos de conservación y la funcionalidad en el sitio (medidas de salud de la biodiversidad).

Considerando la percepción de los encuestados se debe resaltar que los gobiernos locales están por lo común conscientes de las medidas necesarias para cumplir los acuerdos ambientales nacionales y multilaterales. Sin embargo, esa conciencia a 
menudo se restringe a una o dos organizaciones o instituciones, donde en muchos casos no tienen un respaldo para que se puedan ejecutar las iniciativas de conservación y manejo. De manera adicional, los actores claves y la sociedad civil en general conocen sólo acerca de unos pocos esfuerzos de conservación que se podrían desarrollar, pero debido a la voluntad política no se ejecutan.

Las iniciativas para establecer áreas protegidas en el país implican el esfuerzo de individuos, organizaciones nacionales (sector privado y sociedad civil), organizaciones no gubernamentales, instituciones intergubernamentales, por lo que la disposición e identificación de prioridades de los recursos marinos y costeros en Isla Uvita es una prioridad. La necesidad de proponer la Isla como un área marina protegida es de vital importancia, debido a que por las características del sitio se puede considerar como un laboratorio viviente.

Fiske (1992) planteó que las áreas protegidas costeras y marinas se establecen a menudo como respuesta a una crisis existente o una amenaza potencial para la zona costera y, por ello, esas áreas deben abordar los asuntos relacionados con el uso humano intenso y la competencia para tener acceso a los recursos. La presión antrópica y el nulo control sobre el uso de los recursos marinos como la destrucción del arrecife, extracción de peces asociados a ellos y la visitación en la parte insular, entre otras, hace que los actores sociales conscientes del papel que cumplen cada uno de los organismos marinos en el ecosistema, recomienden que se propongan estrategias de conservación para proteger la biodiversidad de la Isla.

La conservación de los recursos bióticos y abióticos del sistema insular de Isla Uvita se genera con la propuesta de estrategias que justifiquen y minimicen las amenazas a los que están siendo sometidos los recursos biológicos y, a la vez, que se propongan escenarios de restauración y del manejo ecológico (Poiani, 2001). En estos procesos hay que incentivar la toma de decisiones sobre asuntos ambientales relacionados con el recurso marino-costero, promoviendo el liderazgo y la participación de los actores claves y la sociedad civil, de manera que puedan convertirse en defensores de la protección de los recursos marinos y costeros.

A la hora que se gesten iniciativas de conservación es importante incorporar a los diferentes sectores claves en el manejo y administración de los recursos marinos y costeros, se debe tener en cuenta que la participación de las comunidades y organizaciones no gubernamentales es decisiva para el manejo y conservación de las áreas marinas protegidas. Los grupos y organizaciones interesados en administrar las áreas protegidas están creciendo constantemente y aportan ventajas 
organizativas y administrativas muy eficientes (Valle et al. 2001).

Es necesario coordinar el manejo de los recursos naturales con todos los actores interesados e incentivar el diálogo con el MINAET, para lograr alguna categoría de conservación que permita proteger los recursos naturales de la Isla. Debe existir un compromiso y el desarrollo de un trabajo conjunto formalizado a través de convenios con instituciones estatales y privadas y la promoción de educación marina y costera, como pilar fundamental para la promoción del compromiso público con la conservación del espacio natural de Uvita.

El manejo participativo de los recursos es un proceso que valora el aprendizaje permanente y la adaptación de las nuevas percepciones a las condiciones cambiantes. Bass et al. (1995) establecen que las comunidades desarrollan un proceso de comprensión del sistema social, natural, económico y local, ya que analizan cómo las acciones políticas impactan sobre esos sistemas.

A continuación se hace una aproximación sobre un posible esquema de manejo que se podría estar desarrollando en la Isla, integrando la participación del sector social. En la Figura 3 se muestra el esquema de la estrategia de manejo propuesta, considerando tres aspectos importantes: la conservación, las líneas estratégicas y la línea de acción principal.

\section{Establecer una organización insti- tucional efectiva}

Se recomienda que esta entidad sea conformada por un miembro o representante de cada una de las diferentes instituciones claves, buscando la integración de conceptos multidisciplinarios para el Manejo Costero Integrado (MCI), de acuerdo con la estructura organizacional que se propone en la Figura 4.

Estas organizaciones deben estar claramente identificadas con los recursos marinos y costeros de la Isla, tener el compromiso con el proceso de responsabilidades, transparencia en sus intereses y acciones, capacidad de gestión en conjunto, disposición a tomar decisiones y acuerdos de consensos y resolver los conflictos primeramente entre las partes, capacitarse en aspectos legales, administrativos, técnicos y financieros, interés por hacer prevalecer el bien común sobre el interés particular.

Esta estructura administrativa debe comenzar labores mediante la promoción de la importancia biológica de la Isla para declararla Área Marina Protegida (AMP), también desarrollar un programa de manejo como punto medular de la administración. Entre sus funciones se citan:

- El manejo y control del recurso económico y humano.

- Establecer y mantener sistemas de control administrativos e inventarios de bienes adquiridos 


\section{Figura 3}



Esquema general de la estrategia de manejo.

General layout of the management strategy. 


\section{Figura 4}



Organigrama de la propuesta administrativa para el manejo de Isla Uvita, Limón.

Organizational chart of the administrative proposal for the management of Uvita Island, Limon. 
como los recibidos en donativos o préstamos.

- Definir y planificar las necesidades financieras de Isla Uvita.

- Seleccionar y reclutar al personal necesario para el control de las actividades en el área.

- Fomentar la participación de los sectores claves para la implementación de un sistema de autofinanciamiento.

- Coordinar todos los estudios para el análisis del desarrollo de infraestructura u otras actividades.

- Tener estrecha relación y coordinación con el MINAET.

- Solicitar constante capacitación para los diversos programas por desarrollar.

- Estrechar relaciones con los encargados del área de gestión ambiental del sector agropecuario.

- Coordinar la producción de material divulgativo de educación y conservación de la biodiversidad de la Isla.

- Implementar la zonificación y regulaciones aprobadas para el turismo, con el apoyo del control, vigilancia y monitoreo, conjuntamente con otras organizaciones.

\section{Declaratoria de Isla Uvita como un AMP}

El área marina de Isla Uvita debe ser declarada como un AMP, para lograr restaurar y proteger el ecosistema natural y la diversidad biológica, para obtener beneficios mutuos de uso y conservación. Mediante el estudio de la biota marina, así como su dinámica de corrientes y formaciones coralinas, se deben establecer los límites de protección, para que estos sean informados, socializados y respetados por los usuarios.

Como punto fundamental se debe trabajar para la creación de un parque marino, donde se establezca la conectividad de la región con las demás áreas marinas del Caribe.

Una forma de conservar los recursos marinos y costeros es mediante la protección integral del área terrestre y marina, donde se garantice un uso sostenible de esta y esté articulada con el elevado valor económico, ecológico y social.

\section{Mapas de zonificación}

Se debe promover una zonificación consensuada a través de diversas reuniones y talleres con los sectores claves, donde se establezcan compromiso y liderazgo por parte de la administración, la cual asumiría la responsabilidad de hacer cumplir la reglamentación establecida para el adecuado funcionamiento del programa de manejo.

Se definirá de qué manera se regula, permite y autoriza el aprovechamiento de los recursos naturales de la Isla, estableciendo para tal efecto los criterios y políticas de conservación, con fundamento en la aplicación de la normativa que se apruebe para tal fin. De conformidad con el componente de 
investigación, aportará las bases, cuotas, límites de uso, métodos y mecanismos de aprovechamiento sustentable de cada tipo de recurso, a través de la evaluación y análisis de los usos y costumbres locales. Por lo tanto, los propósitos de la zonificación corresponden:

- Fomentar el uso y aprovechamiento sustentable de los recursos naturales, regulando las actividades turísticas, pesqueras y propiciando la participación social.

- Asegurar, para beneficio de los pobladores locales y usuarios, la persistencia de los recursos naturales de la Isla, así como de los atractivos que permiten el uso turístico por la belleza escénica.

- Establecer y regular los límites máximos permisibles de uso y monitorear las actividades acuático-recreativas, con base en la zonificación.

- Regular la pesca comercial, de autoconsumo, para lograr un uso sustentable de los recursos pesqueros.

Hay que establecer e implementar la zonificación y regulaciones para que sean aprobadas a favor de la conservación y manejo de los recursos, estableciendo un apoyo de control, vigilancia y monitoreo, mediante la participación de locales voluntarios y entidades gubernamentales.

\section{Investigación y monitoreo}

La investigación y el monitoreo son fundamentales para la retroali- mentación y ajuste de todas las actividades. El monitoreo está orientado a conocer los cambios en los sistemas ecológicos y las poblaciones de especies prioritarias (impacto), reconoce prácticas de manejo del recurso, las actividades que se desarrollan administrativamente y aquellas que deben estar contenidas en el programa de administración (desempeño).

Debe ser enfocado a la generación y difusión de información técnica y científica, que sustente la gestión del buen funcionamiento no sólo del área, sino generar información y herramientas para la aplicación en otras regiones, estableciéndose prioridades para el cumplimiento de los objetivos.

Es primordial generar herramientas de conservación y seguimiento, promoviendo la coordinación y participación de los actores claves e incentivar un proceso participativo, donde las instituciones gubernamentales, no gubernamentales, comunidades y otros sectores se involucren en dicho trabajo.

Esta actividad es indispensable para determinar el grado de afectación de los ecosistemas marinos y costeros de la Isla, tomando en cuenta la conservación de los recursos naturales y la divulgación del sitio como importante destino turístico. Se deben establecer sistemas adecuados para controlar los desechos sólidos, así como la disposición de los otros desechos propios de la actividad.

Para un mejor conocimiento del ecosistema se debe promover el diseño de un sistema de información, 
considerándose los indicadores ecológicos, la definición de las necesidades del área y las prioridades de investigación.

El conocimiento de las condiciones ecológicas, sociales y económicas de la Isla, conjuntamente con los usos y presiones ejercidas, permitirán la identificación y jerarquización de la problemática existente, lo que contribuirá a determinar las reglas administrativas para la conservación y aprovechamiento sostenible de sus recursos. Por otra parte, definirán los mecanismos de participación de los diversos sectores, de tal forma que sean factibles el cumplimiento de los objetivos de esta propuesta.

Entre los aspectos recomendados en investigación y monitoreo están:

- Estudios de la calidad biológica y química del agua.

- Estudio de la biodiversidad de los ecosistemas marinos de la Isla.

- Clasificación del hábitat, desarrollar un sistema de información geográfica del arrecife donde se generen indicadores para el manejo.

- Estudios sobre reclutamiento de peces juveniles, análisis espacial sobre las comunidades de peces de arrecife y la relación con el hábitat de manera periódica.

- Estudios sobre la situación o estado de la pesca en los alrededores de la Isla.

- Inventario de moluscos y crustáceos.

- Monitoreo de las rutas a las zonas de alimentación, reproducción y migración de las tortugas carey que anidan en la Isla.

- Estudios poblacionales en diferentes épocas para conocer la densidad y composición poblacional de aves residentes y migratorias marinas.

- Estudio de los patrones del uso de hábitat y explotación de recursos por las aves residentes y migratorias en diferentes partes del año según las densidades.

- Estudios de aves marinas.

- Estudios e investigación de los murciélagos.

\section{Educación y capacitación marina y costera}

Debido a que las actividades turísticas y de pesca sin control son factores que influyen negativamente en el ecosistema marino y costero, se considera primordial educar, capacitar y difundir información dirigida a la población local y a todos los sectores involucrados en dichas actividades. Hay que sensibilizar a los operadores turísticos sobre la importancia de la formación continua de mejores prácticas turísticas enfocadas en el uso, educación y conservación.

Se pretende crear un ambiente de conocimiento general y sensibilización sobre la fragilidad del ecosistema marino, con el fin de motivar entre la población en general y los prestadores de servicios la participación en campañas de protección ambiental. 
Buscar que las comunidades costeras limonenses se integren a la conservación y manejo de los recursos biológicos de la Isla, es uno de los principales objetivos y encontrar la manera que se beneficien de ello, partiendo de que existe una actitud positiva de las comunidades locales en relación con la necesidad de contribuir al adecuado manejo del área costera.

Se deben tener en cuenta las siguientes recomendaciones para esta línea estratégica de capacitación y educación:

- Apoyo a personas líderes y emprendedoras en sus iniciativas conservacionistas y otras de beneficio para las comunidades.

- Fortalecimiento de la organización comunal (patronatos, asociaciones de guías turísticos, entre otros) y sus líderes.

- El respeto y conservación de las costumbres y tradiciones afrocaribeñas.

- Tanto en los individuos como en las organizaciones, se debe incentivar el desarrollo de proyectos realistas que disminuyan la presión sobre el área protegida, entre las comunidades costeras.

- Promover la participación de organizaciones gubernamentales y no gubernamentales.

\section{Recreación y uso}

La ubicación y riqueza biológica de la Isla, la posiciona como un sitio que ofrece experiencias turísticas especiales de calidad, comprometida con la conservación de los recursos y con la satisfacción de los turistas.

La entidad administrativa de la Isla debe coordinar con empresas, turistas y pobladores, los permisos de operación dentro del área, con el fin de que se controlen todas las actividades en el sitio para prevenir el deterioro del ecosistema.

Se debe informar a los turistas, a través de charlas de inducción, sobre las políticas de uso y la normativa de acatamiento de la zonificación, así como la disposición apropiada de desechos y el respeto a las diversas formas de vida.

Consideraciones por desarrollar en la actividad turística:

- Las actividades turísticas deben desarrollarse únicamente en los sitios autorizados y asignados para cada experiencia, tales como buceo organizado, "snorkeling", playa, caminatas demarcadas e interpretadas, experiencias culturales y mantener la calidad de estas experiencias y servicios.

- Los operadores turísticos deben facilitar una operación ordenada, participativa y responsable por los recursos y la experiencia de los turistas.

- El impacto a los recursos marino-costeros debe ser mínimo y localizado, garantizando algunas áreas sin uso como hábitats frágiles.

- Se debe promover el desarrollo de la mínima infraestructura para sa- 
tisfacer las necesidades básicas a los visitantes, letrinas y duchas.

- Promover oportunidades para que personas de comunidades locales se organicen en microempresas que provean servicios organizados de calidad a los turistas.

Entre algunas experiencias que se pueden llevar a cabo pueden ser: caminatas, buceo, área de baño, surf, experiencia cultural.

Recomendaciones para el ordenamiento del desarrollo de actividades en la Isla:

- Divulgación de cómo debe ser el comportamiento del visitante, según la zonificación, restricciones de actividades que comprometan la integridad del recurso, la calidad de la experiencia de los visitantes y seguridad.

- Estructuración de la operación y control de calidad mediante una organización local como una asociación de turismo.

- Promoción del área como una operación turística de un día.

- Utilización de guías, capitanes locales autorizados por el ente administrador, donde haya una inscripción ante la entidad administradora para tal fin.

- Uso de un sistema de reservaciones y programación de visitas para el control de ingreso.

- Construcción y mantenimiento de senderos y muelles que den segu- ridad a los turistas y reduzcan el impacto al ambiente.

- No se debe promover la construcción de infraestructura turística en el área.

- Monitoreo del grado de satisfacción $\mathrm{y}$ de las conductas de los turistas.

Toda actividad turística debe ser centrada en el ordenamiento de la operación, tomando en cuenta los acuerdos con los representantes de los grupos locales de interés y la promoción del uso responsable con conciencia y respeto al sitio.

\section{BIBLIOGRAFIA}

Bass, S. M. J., Dalal-Clayton, D. B. and Pretty, J. (1995). Participation in Strategies of Sustainable. Development, Environmental Planning Issues, no 7, International Institute for Environment and Development, London. No. 7. $118 \mathrm{p}$.

Baumgartner, J. (2001). Planificación para la Conservación de Sitios PROARCA/COSTAS. Un esquema para desarrollar y medir el impacto de estrategias efectivas de conservación de la biodiversidad. $50 \mathrm{p}$.

BID-Banco Interamericano de Desarrollo. (1998). Manejo de los recursos costeros y marinos en América Latina y el Caribe. Departamento de Desarrollo Sostenible. División de Medio Ambiente. 
Washington, D.C. No. ENV-128. $46 \mathrm{p}$.

Comisión Interinstitucional de la Zona Económica Exclusiva de Costa Rica. (2008). Estrategia Nacional para la Gestión Integral de los Recursos Marinos y Costeros de Costa Rica. San José, Costa Rica. 74 p.

Fiske, S. (1992). Sociocultural aspects of establishing marine protected areas. Ocean Coas. Manage., 18, 25-46.

Gómez Barrantes, M. (1998). Elementos de estadística descriptiva. San José, C.R.: EUNED. 542 p.

Gunderson, L. H. and Pritchard, L. Jr. (2002). Resilience and the Behaviour of Large-Scale Ecosystems. Washington DC: Island Press. 257 p.

Hernández Sampieri, R., Fernández Collado, C. y Baptista Lucio, P. (2006). Metodología de la investigación. México: McGraw Hill. 850 p.

INEC-Instituto Nacional de Estadística y Censo (2004). Documento Metodológico Censo Nacional de Población 2000. 33 p.
Ochoa, E. (1998). Manual para un Taller Comunitario. Centro de Recursos Costeros de la Universidad de Rhode Island. PROARCA-Costas, TNC, WWF, CRC/ URI. USAID/G-CAP. Guatemala. $33 \mathrm{p}$.

Poiani, K. (2001). Paisajes funcionales y la conservación de la biodiversidad. TNC. $12 \mathrm{p}$.

PROARCA (Programa Ambiental Regional para Centroamérica). (1998). Cuaderno de Trabajo para Preparar un Plan de Manejo Costero Integrado. TNC, WWF, University of Rhode Island-CRC. Guatemala. 42 p.

Valle, L., Fonseca, A., Paiz, Y., Brown, M. y Secaira, F. (2001). Evaluaciones Ecológicas Rápidas. Herramienta de Manejo Costero Integrado para el planificador. PROARCA / COSTAS. 84p. Windevoxhel, N. (2001). Guía para la planificación participativa en áreas marino costeras protegidas. Guatemala: PROARCA/ Costas. $56 \mathrm{p}$. 